\title{
A Combined Nulling and Imaging Pupil-Plane Beam-Combiner for DARWIN
}

\author{
Ron P. H. Haaksman ${ }^{\text {a }}$, Cor P. de Vries ${ }^{\text {a }}$, Jan-Willem den Herder ${ }^{\text {a }}$, L. L. A. Vosteen ${ }^{\text {b }}$, Henk \\ Bokhove $^{b}$, Arjan L. Mieremet ${ }^{c}$ \\ a) SRON Netherlands Institute for Space Research, Sorbonnelaan 2, 3584 CA Utrecht, The Netherlands \\ b) TNO-S\&I, Stieltjesweg 1, 2628 CK Delft, The Netherlands \\ c) cosine Research B.V., Niels Bohrweg 11, 2333 CA Leiden, The Netherlands
}

\begin{abstract}
The primary goal of DARWIN is to detect earth-like extrasolar planets and to search for biomarkers. This is achieved by means of nulling interferometry, using three free-flying telescopes and a Beam-Combiner (BC) hub. DARWIN will be able to perform astrophysical imaging with high spectral and spatial resolution. Should one of Darwin's telescope flyers fail, then Darwin's capability of detecting earth-sized exo-planets is dramatically reduced. However, with only two telescopes the imaging mode can continue operating with minimal performance degradation, thus ensuring mission success.

This work describes a trade-off study between four conceptual three-beam BC's, that are capable of performing both as a nuller and as an imager. A proposed breadboard design will demonstrate end-to-end Fringe-Tracking (FT) and Optical Path-Length (OPL) control. The BC concept is based on a pupil-plane (Michelson) beam combination scheme. Pupil-plane imaging BC's offer a large overlap in terms of optical layout with the nulling $\mathrm{BC}$ concept, making it possible to develop a combined nulling- and imaging BC. This means that a reduced number of optical components can be used compared to a scheme with separate BC's. The BC concept inherently compensates for unequal OPL's, which in ground-based interferometers is compensated for by long stroke Optical Delay Lines (ODL's).
\end{abstract}

Keywords: DARWIN, Stellar interferometry, Planet detection, Beam-Combiner (BC), Nulling/Imaging.

\section{Introduction}

Nulling interferometry is a powerful technique to directly detect extra-solar planets by canceling the light of its nearby star. The implementation of imaging capabilities in addition to the nulling mode is highly advantageous for the DARWIN mission. In the case of loss of one of DARWIN's telescopes, no internal modulation can be performed (internal modulation is the capability of the interferometer system to swap two transmission maps in the nulling mode for correction of background signals and detector drift). This causes a significant drop in Signal-to-Noise Ratio (SNR), effectively rendering the nulling mode useless. However, imaging can still be performed with only two telescope flyers.

By combining a nulling and an imaging mode into one $\mathrm{BC}$ it is possible to reduce the number of optical components. In this paper a trade-off is made between four proposed concepts for the Darwin three-beam nulling/imaging BC. Only concepts are studied which meet the prime science requirements such as spectral resolution, spatial resolution, imaging sensitivity and baseline range [1]. The different concepts were compared using a set of well defined criteria as will be explained in the text.

The analysis of the cophasing optics for the imaging mode and the optical design of a dedicated spectrograph goes beyond the scope of this work and is therefore not addressed. End-to-end FT is the measurement of OPL's up to the interferometric beam combination point, i.e. OPL measurement is done over the full length of the optical train. With a proposed breadboard the most critical aspect can be tested in a dynamical environment, i.e. end-to-end FT. 
The layout of this paper is as follows. Section two will present the criteria that were used in the trade-off study. In section three the four BC concepts are presented and discussed. The trade-off between the concepts is done in section four and the proposed concept breadboard design is presented in the final section.

\section{Trade-Off Criteria}

Four selected BC concepts were compared in this work. The following trade-off criteria were used for concept comparison, whereby concepts are preferred to score high for criteria such as optical throughput, modulation efficiency and Field-of-View (FoV):

\section{Optical Throughput}

The throughput, $\eta$, directly influences the integration time needed to obtain a required SNR for planet detection. In this work the optical throughput is defined as follows:

$$
\eta=\frac{I_{\text {det. }}}{I_{\text {tel. }}}
$$

where $I_{\text {det. }}$ and $I_{\text {tel. }}$ are the total intensities on the FT or nulling detectors and the total intensity collected by the telescopes respectively. A throughput of $100 \%$ indicates that all the light collected by the telescope flyers ends up on the detectors. The throughput assumes no optical loss at optical components.

\section{Modulation Efficiency}

The modulation efficiency for a given array configuration, $\varepsilon$, is a measure of how efficiently a nulling configuration generates a modulated planet output, relative to the total collecting area. The integration time to obtain a required SNR for planet detection is directly related to the modulation efficiency. The modulation efficiency is defined as:

$$
\varepsilon=\frac{I_{\text {constr. }}}{I_{\text {tel. }}}
$$

where $I_{\text {constr. }}$ is the maximum constructive intensity of an off-axis planet. $100 \%$ modulation efficiency indicates that all the collected light is used for generating a planet signal.

\section{Field of View}

In contrast to the unvignetted FoV, the interferometric FoV is defined as the field angle over which the interferometer is capable of producing interferometric fringes. It is an important criterion for the imaging mode of the interferometer, since a large FoV allows imaging of extended astrophysical objects. The FoV of the BC should at least cover a few Point Spread Functions (PSF's). In the trade-off it is tested whether the concept is capable of producing a FoV of a few PSF's or not, indicated with a Yes or a No respectively.

\section{Failure Mode}

Failure mode indicates whether the interferometer can operate if one of the three telescope flyer beams fails and indicates the collected amount of radiation by the remaining telescopes that can be used for imaging. A failure mode of $100 \%$ indicates that all the light collected by the remaining two telescopes is used for imaging.

\section{Optical Path Symmetry}

Deep nulling is only possible if the optical paths within the BC are fully symmetric. Asymmetric optical paths may introduce issues like chromatic phase differences and differential polarization, which have a detrimental effect on fringe contrast. 
Optical Path Length control

OPL control will probably prove to be a significant issue since slow beam intensity and phase drifts could generate mock planet signals. These mock signals can be calibrated out utilizing end-to-end FT since, with this FT technique, OPL measurements are done over the full length of the optical train. Hence, only one control loop is necessary. Moreover, end-to-end FT can be done in the science wavelength band. However, not all BC concepts are suitable for the implementation of end-to-end FT, necessitating the use of multiple control loops, which then reduces the FT accuracy, and increases system complexity.

\section{Fringe Type}

This item indicates whether the concept utilizes OPL scanning to obtain temporal fringes or produces spatial fringes across the detector surface. With spatial fringes no OPL scanning is necessary. However, the issue with spatial fringes is how to yield spectral information from the fringes (a technique such as image slicing can be utilized, but this would increase the complexity of the detection part). Yielding spectral information from the interferometric fringes is not an issue with temporal fringes, since an arbitrary direction across the detector surface can be used for fringe dispersion. A more sophisticated FT technique is where the fringe amplitude and phase are obtained by electronic addition/subtraction of detector output signals, whereby some of the signals are appropriately shifted in phase. This method is highly advantageous since it does not require OPL scanning and spectral information is readily available by fringe dispersion. An overview of the trade-off criteria can be found in Table 1.

Table 1: List of concept trade-off criteria for the nulling and the imaging mode of the $\mathrm{BC}$ concepts.

\begin{tabular}{|ll|cc|}
\hline Trade-off criteria & & Nulling & Imaging \\
\hline Optical throughput & {$[\%]$} & $\geq 35(100$ idealized $)$ & 100 \\
\hline Modulation efficiency & {$[\%]$} & $\geq 50$ & $(\mathrm{~N} / \mathrm{A})$ \\
\hline Field of View & {$[\mathrm{arcsec}]$} & $\geq 0.5$ & $\geq(0.5-1)$ \\
\hline Failure mode & & $($ Yes/No) & $($ Yes/No) \\
\hline Optical path symmetry & & (Yes/No) & (Yes/No) \\
Optical Path Length control & & \multicolumn{2}{|c|}{$\begin{array}{c}\text { End-to-end FT, or } \\
\text { Multiple control loops }\end{array}$} \\
Fringe type & & \multicolumn{2}{|c|}{$\begin{array}{c}\text { Temporal fringes, or } \\
\text { Spatial fringes, or }\end{array}$} \\
& & \multicolumn{2}{|c|}{ Electronic fringe detection } \\
\hline
\end{tabular}

$\mathrm{N} / \mathrm{A}=$ Not Available for the imaging mode. 


\section{Beam-Combiner Concepts}

In this section a detailed description of the four selected BC concepts for the trade-off study will be given, knowing the:

1. Image-plane $\mathrm{BC}$

2. Modified TTN + BC

3. Cosine $\mathrm{BC}$

4. TNO BC

The main advantages and disadvantages of each concept will be addressed for the concept trade-off.

In general, with interferometric beam combination distinction can be made between coaxial and multiaxial beam combination as is illustrated in Figure 1.

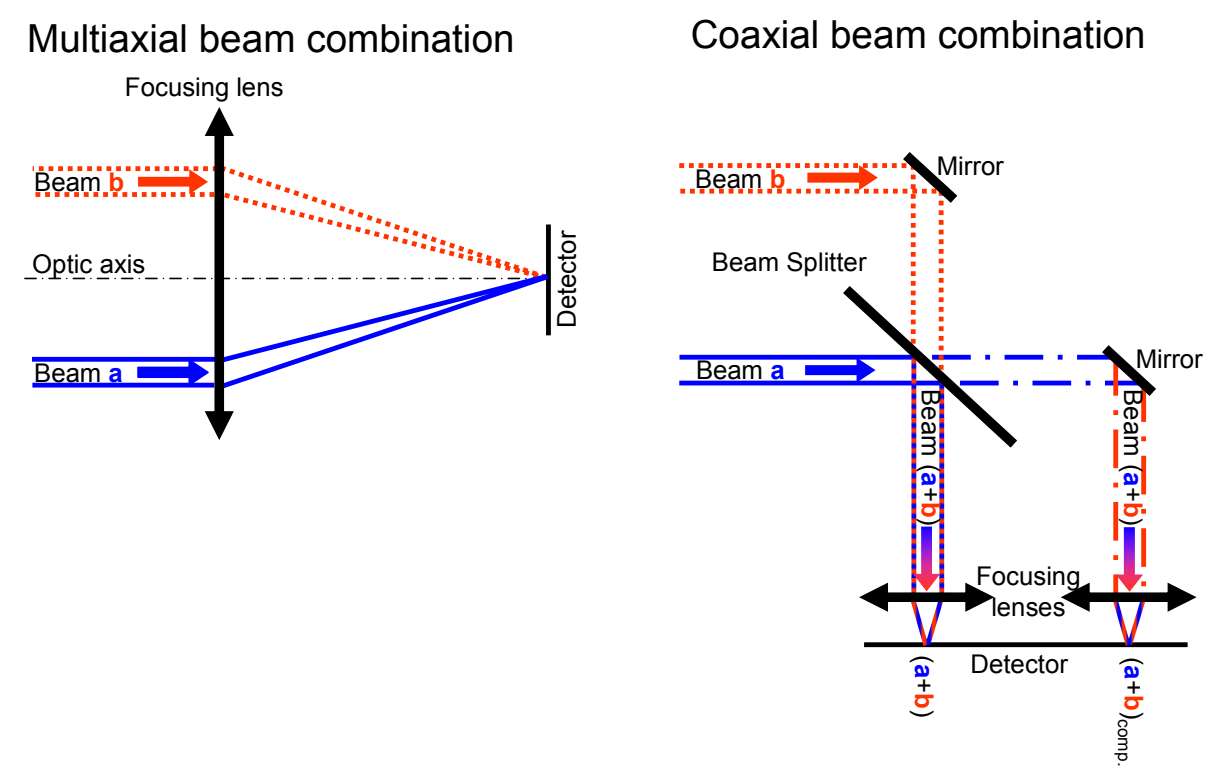

Figure 1: Definition of multiaxial and coaxial beam combination.

It has to be mentioned that with multiaxial beam combination there is an issue with beam coupling into a Single-Mode Fiber (SMF). Although the authors are aware of this it was not addressed in this work, and will be dealt with in further work. 


\subsection{Image-plane Beam-Combiner}

The simplest in concept is combining beams in the image-plane. This concept is basically a masked aperture and provides an inherently large FoV when the interferometer complies with the homothetic condition (i.e. the exit pupils are an exact scaled replica of the input pupils). Two possibilities exist to comply with the homothetic condition, knowing the variable pupil configuration whereby the exit pupils are translated (mirrors M10 in Figure 2) and the variable pupil magnification whereby the exit pupils are magnified [2]. A maximum $100 \%$ modulation efficiency is obtained with the image-plane BC.

Beam combination in the image-plane generates spatial fringes across the detector surface, eliminating the need for OPL fringe scanning. A major disadvantage of combining beams in the image-plane is that it does not allow for internal modulation, since the $\mathrm{BC}$ only provides one interferometric output (no complementary output). Moreover, relatively large imaging optics are necessary in order to obey the homothetic condition, and gaining spectral information from spatial fringes by image slicing would increase the system complexity. The image-plane BC concept is fully telescope failure safe.

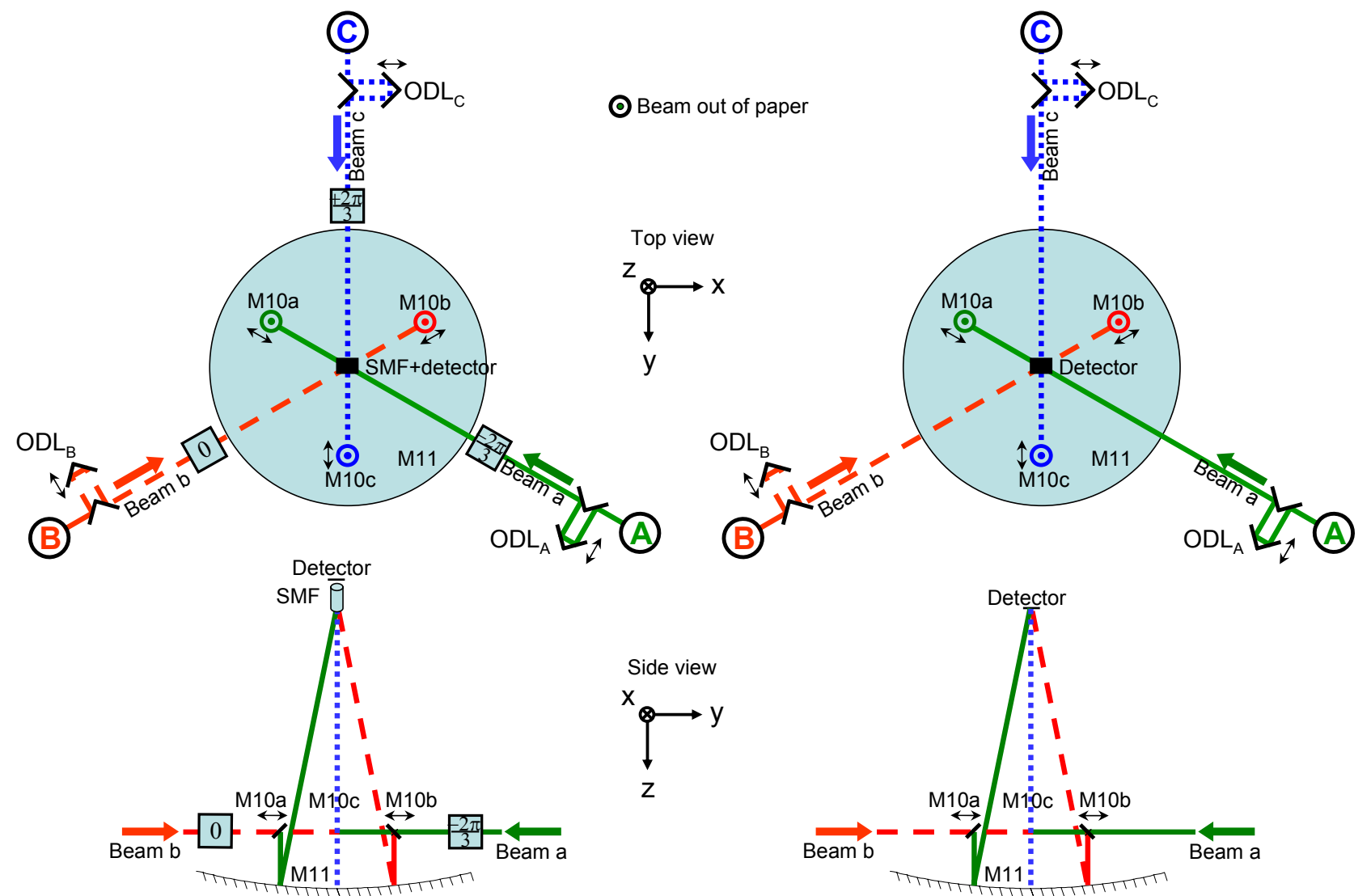

Figure 2: Schematic of the homothetic image-plane BC concept in the nulling mode (left) and the imaging mode (right). In the imaging mode the phase shifters are bypassed and the SMF is removed. 


\subsection{Modified TTN+ Beam-Combiner}

This is a modified concept to the one that was put forward by ESA [3]. BC concepts found in literature have optical layouts with inherently unequal OPL's for the combined beams in the BC. This can easily be compensated for with long stroke ODL's that are necessary in ground-based stellar interferometers since they have to compensate for earth rotation as well. However, long stroke ODL's are highly impractical in space-based systems. The modified TTN+ BC concept has effectively eliminated the unequal OPL's by folding the beams inwards, albeit at the cost of two extra reflections per beam (see Figure 3).

The BC layout is based on a Mach-Zehnder (MZ) interferometer with a reciprocal Beam Splitter (BS) pair for symmetry reasons of the nulled interferometric output. The three beams coming from the telescope flyers enter the $\mathrm{BC}$ from the top left corner. Each beam is initially amplitude split into two beams of equal intensity at a first BS (BS1). The six beams are subsequently cross combined at a second BS (BS2). For the imaging mode the six interferometric beam pairs are then focused onto the surface of an imaging detector. Applying OPL scanning produces temporal fringes on the imaging detector surface. The $\pi / 2$ and the $\pm 2 \pi / 3$ phase shifters are necessary for nulling. The $\pm 2 \pi / 3$ phase shifters are bypassed in the imaging mode, while the $\pi / 2$ phase shifters are incorporated in the imaging mode. However, applying $\pi / 2$ phase shifts in the imaging mode has no significant influence on the imaging performance. The $\pi / 2$ phase shift can be compensated for with the ODL's in each of the three beams. For symmetry reasons 0 radian phase shifters are also incorporated. In the nulling mode the six interferometric beam pairs, generated by cross combination at BS2, are further combined by focusing them into the guiding core of an SMF where the nulling takes place. The interferometric beam $(\mathbf{a}+\mathbf{b}+\mathbf{c})$ and its complementary beam $(\mathbf{a}+\mathbf{b}+\mathbf{c})_{\text {comp }}$ are subsequently focused onto the surface of the nulling detector, where both beams produce spatial fringes (due to the multiaxial beam combination). Notice that the beams reflect off the mirrors and BS's at near normal incidence ( $\sim 10$ degrees). This was done in order to minimize possible unwanted polarization effects.

The BC can be switched back and forth between the nulling mode (Figure 3 top) and the imaging mode (Figure 3 bottom) by flipping four fold mirrors within the BC (mirrors M23ab, M23ac and M23bc). The temporal fringes on the imaging detector surface, can be dispersed in an arbitrary direction across the detector surface yielding spectral information. The spatial fringes can be dispersed as well, since the spectral resolution necessary for the nulling mode is much lower compared to the imaging mode (on the order of 50 instead of 300) so no significant fringe smearing would occur. The concept yields a relatively high modulation efficiency of $93 \%$. The major disadvantage of this $\mathrm{BC}$ concept is that it cannot support end-to-end FT. Should one of the three telescopes fail, then 50 percent of the light will be lost from the interferometric channels and will end up in the photometric channels. This can be fully compensated for by manufacturing BS1 with different coated areas on one of its surfaces. Together with a necessary linear translation of BS1 along the $\mathrm{X}$-axis (see Figure 3) in case of single telescope failure, so that the beams coming from the two remaining telescope flyers impinge on these coated areas. It has to be mentioned that this increases the system complexity which was not taken into account in the concept trade-off described in the next section. 

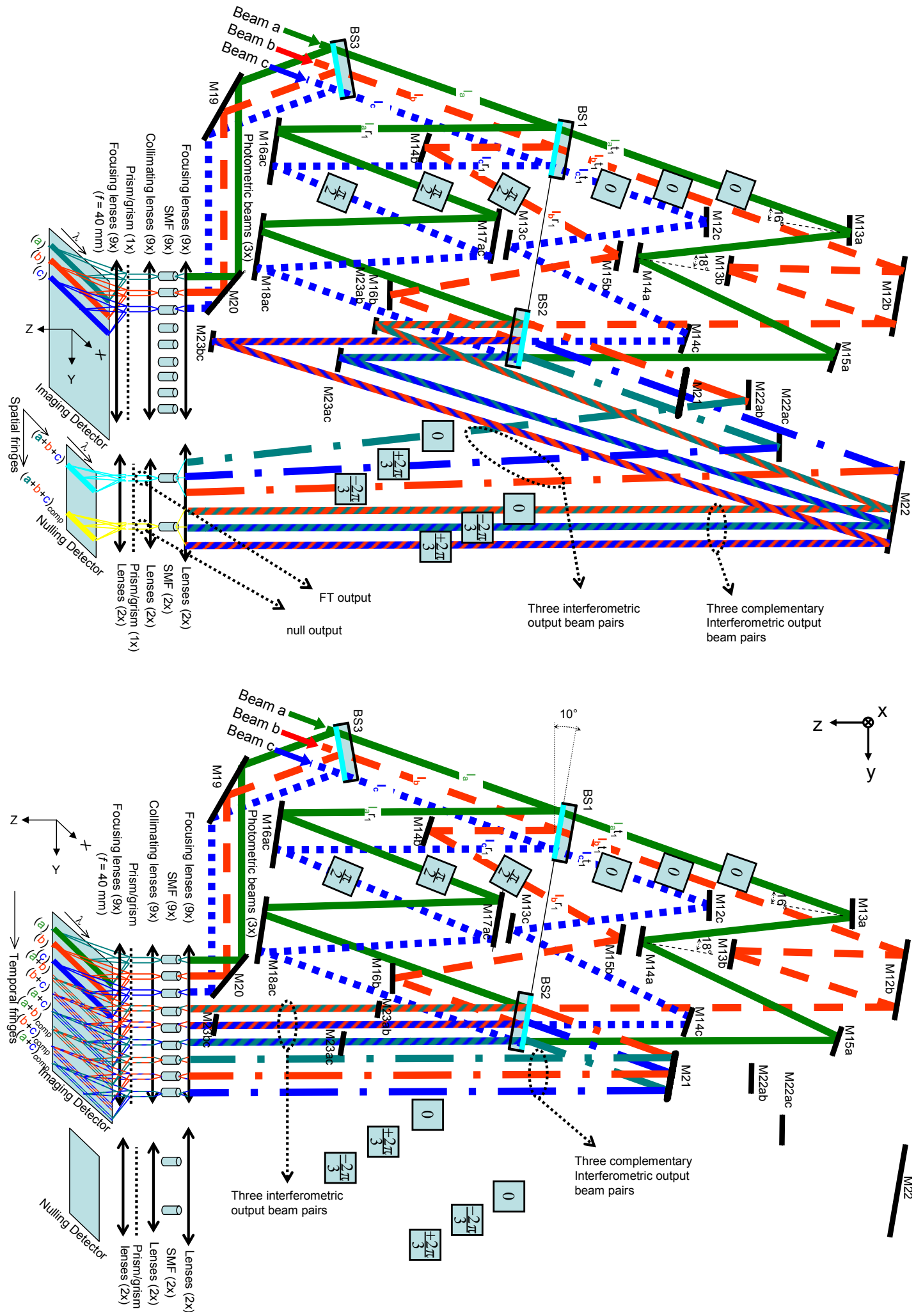

Figure 3: Schematic of the modified TTN + BC in the nulling mode (top) and in the imaging mode (bottom). The three telescope beams are fed into the $\mathrm{BC}$ from the top left corner. 


\subsection{Cosine Beam-Combiner}

This concept is similar to the modified TTN $+\mathrm{BC}$ except for the nulling mode where the $\pm 2 \pi / 3$ radian phase shifters and the second row of BS's are removed from the $\mathrm{BC}$ utilizing six fold mirrors. The interferometer has a circle symmetrical layout as depicted in Figure 4. The downside of this concept, like with the modified TTN+ BC concept, is that it cannot support end-to-end FT. Furthermore, it produces a relatively low modulation efficiency of $50 \%$. Fringe detection is done by OPL scanning, hence the generated temporal fringes can be dispersed across the detector surface for spectral information.
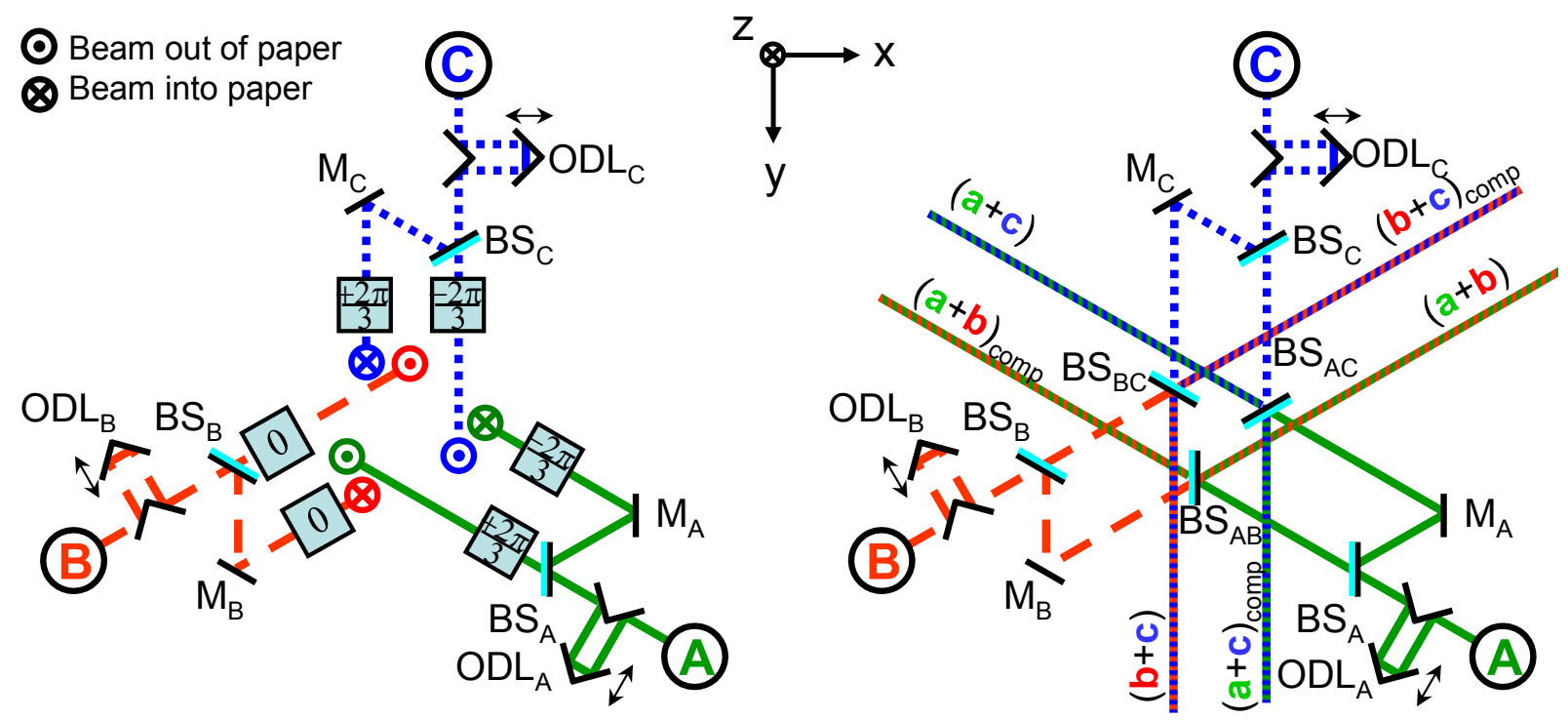

Figure 4: Schematic of the cosine $\mathrm{BC}$ concept in the nulling mode (left) and the imaging mode (right).

In the nulling mode the beams are directed along the positive and the negative $\mathrm{Z}$-axis and are subsequently multiaxially focused into the core of an SMF. In the imaging mode the beams are coaxially combined at three cross combining BS's $\left(\mathrm{BS}_{\mathrm{AB}}, \mathrm{BS}_{\mathrm{BC}}\right.$ and $\left.\mathrm{BS}_{\mathrm{AC}}\right)$. Note that in the nulling mode the cross combining BS's are bypassed by utilizing six fold mirrors that flip into place when operating in the nulling mode, and that in the imaging mode the six phase shifters are bypassed. 


\subsection{TNO Beam-Combiner}

With the TNO BC beam combination for both the nulling and the imaging mode takes place coaxially (see Figure 5). Each beam is initially split into two beams of equal intensity at $\mathrm{BS}_{\mathrm{A}}, \mathrm{BS}_{\mathrm{B}}$ and $\mathrm{BS}_{\mathrm{C}}$. Both beams are subsequently fed into core 1 and core 2. Both cores generate a null output and three FT outputs which are used for driving the control loops (i.e. the ODL's) and for generating the two imaging outputs. Switching between the nulling and the imaging mode is done electronically with the detector output signals:

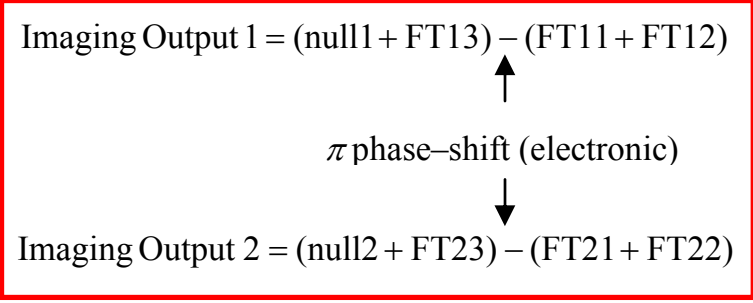

This means that, although the beams are combined coaxially, no OPL scanning is necessary for fringe detection. The BC allows for end-to-end FT to be fully implemented and fringe dispersion yields the necessary spectral information. A further advantage of the TNO BC concept is that it can perform nulling and imaging simultaneously. Furthermore, the $\mathrm{BC}$ is inherently telescope failure safe. A significant disadvantage of the TNO concept is its relatively low $37.5 \%$ modulation efficiency.

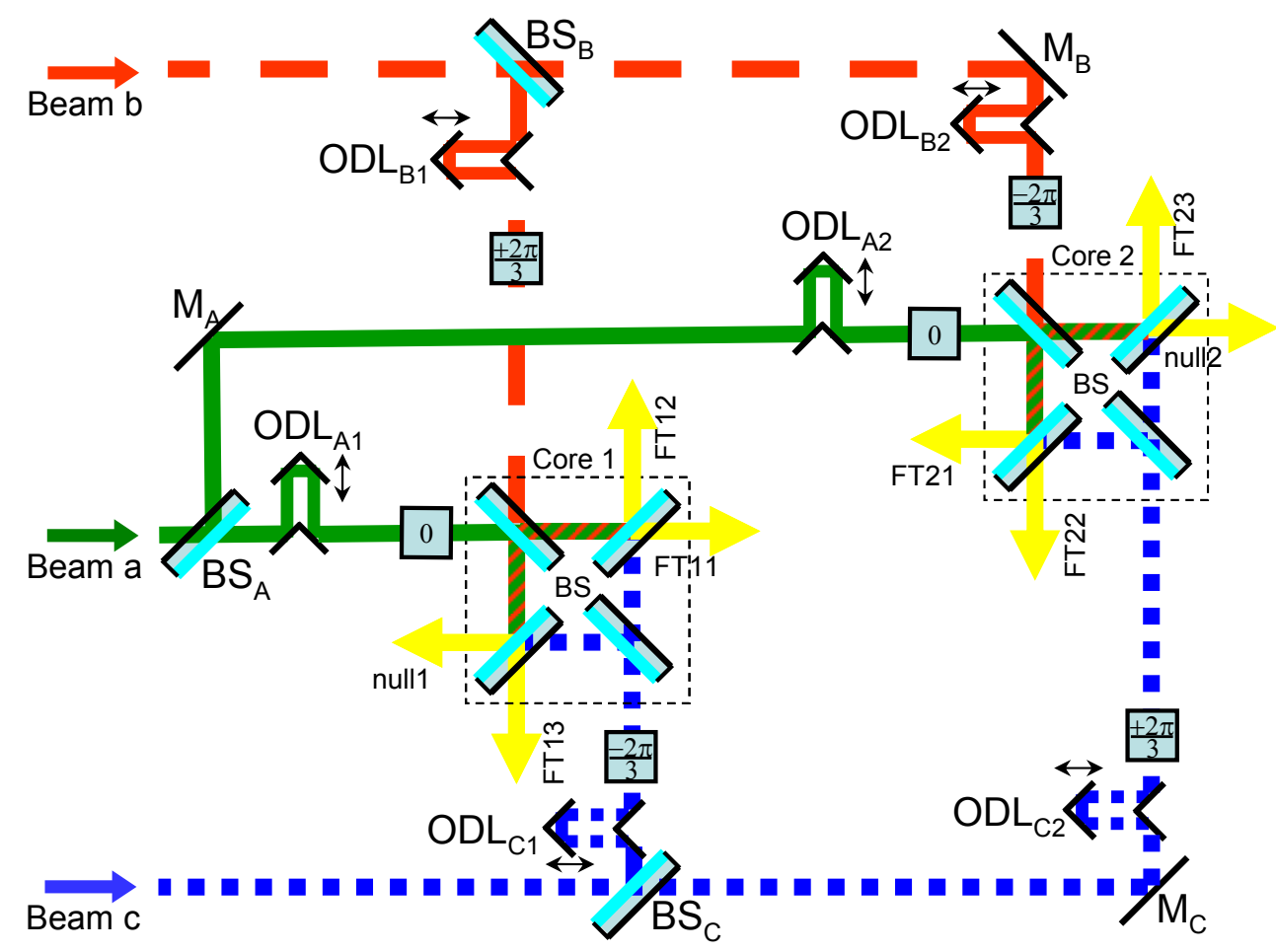

Figure 5: Schematic of the TNO BC concept.

Note that the null1 and the null 2 outputs are the only two outputs capable of producing deep nulls, due to the fact that all three beams combined within both cores, that end up in the nulled outputs, have experienced the same number of BS reflections and also have propagated through the BS's substrate the same number of times, making the null outputs symmetrical outputs. 


\section{Concept Trade-Off}

A trade-off between the four BC concepts presented in the previous section can now be done. The selected trade-off criteria are listed in Table 2.

Table 2: Concept trade-off.

\begin{tabular}{|c|c|c|c|c|c|c|}
\hline & Trade-off criteria & & $\begin{array}{c}\text { Image-plane } \\
\text { BC }\end{array}$ & $\begin{array}{c}\text { Modified } \\
\text { TTN+ BC }\end{array}$ & $\begin{array}{c}\text { Cosine } \\
\text { BC }\end{array}$ & $\begin{array}{c}\text { TNO } \\
\text { BC }\end{array}$ \\
\hline \multirow{8}{*}{ 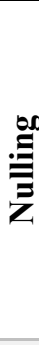 } & Beam combination & & Multiaxial & Multiaxial & Multiaxial & Coaxial \\
\hline & Optical throughput & {$[\%]$} & 100 & 100 & 100 & 100 \\
\hline & Modulation efficiency & {$[\%]$} & 100 & 93 & 50 & 37.5 \\
\hline & Field of View & {$[\mathrm{Y} / \mathrm{N}]$} & Yes $^{(+)}$ & Yes & Yes & Yes \\
\hline & Failure mode & {$[\%]$} & 0 & 0 & 0 & 0 \\
\hline & Optical path symmetry & {$[\mathrm{Y} / \mathrm{N}]$} & Yes & Yes & Yes & Yes \\
\hline & End-to-end FT & {$[\mathrm{Y} / \mathrm{N}]$} & No & No & No & Yes \\
\hline & Fringe type & & Spatial & Spatial & Spatial & Electronic \\
\hline \multirow{8}{*}{ 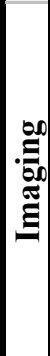 } & Beam combination & & Multiaxial & Coaxial & Coaxial & Coaxial \\
\hline & Optical throughput & {$[\%]$} & 100 & 100 & 100 & 100 \\
\hline & Modulation efficiency & {$[\%]$} & (N/A) & $(\mathrm{N} / \mathrm{A})$ & $(\mathrm{N} / \mathrm{A})$ & (N/A) \\
\hline & Field of View & {$[\mathrm{Y} / \mathrm{N}]$} & $\mathrm{Yes}^{(+)}$ & Yes & Yes & Yes \\
\hline & Failure mode & {$[\%]$} & 100 & 50 & 50 & 100 \\
\hline & Optical path symmetry & {$[\mathrm{Y} / \mathrm{N}]$} & Yes & Yes & Yes & Yes \\
\hline & End-to-end FT & {$[\mathrm{Y} / \mathrm{N}]$} & Yes & Yes & Yes & Yes \\
\hline & Fringe type & & Spatial & Temporal & Temporal & Electronic \\
\hline
\end{tabular}

$(+)$ Fullfils this criterion by a large margin.

End-to-end FT is a much preferred option, since OPL drifts are anticipated to be a significant issue. The only concept with the capability of implementing end-to-end FT is the TNO BC. The only disadvantage of the TNO concept is the relatively low modulation efficiency. There is, however, scope to improve upon this figure and this work has already started. Furthermore, the $\mathrm{BC}$ scheme has scope for delivering the required spectral information with a resolution on the order of 300 . 


\section{The concept breadboard}

The principle aim of the breadboard design is to demonstrate the feasibility of end-to-end FT and OPL control with a TNO-based three-beam BC in air at room temperature. Possible polarization issues will be addressed as well. The breadboard will be operated at mid-Infrared wavelengths with a center wavelength of $(2-4)$ micron and a $(10-20)$ percent bandwidth. The beams will have a cross sectional diameter of approximately $(15-20) \mathrm{mm}$.

The proposed breadboard design is shown in Figure 6. Testing of internal modulation can be achieved by either swapping of the $\pm 2 \pi / 3$ radian phase shifters, or by swapping of the telescope beams. In this particular design internal modulation is performed by means of beam swap optics. Implementing beam swap optics is much easier to accomplish than changing the sign of the $\pm 2 \pi / 3$ radian phase shift applied by the four phase shifters.

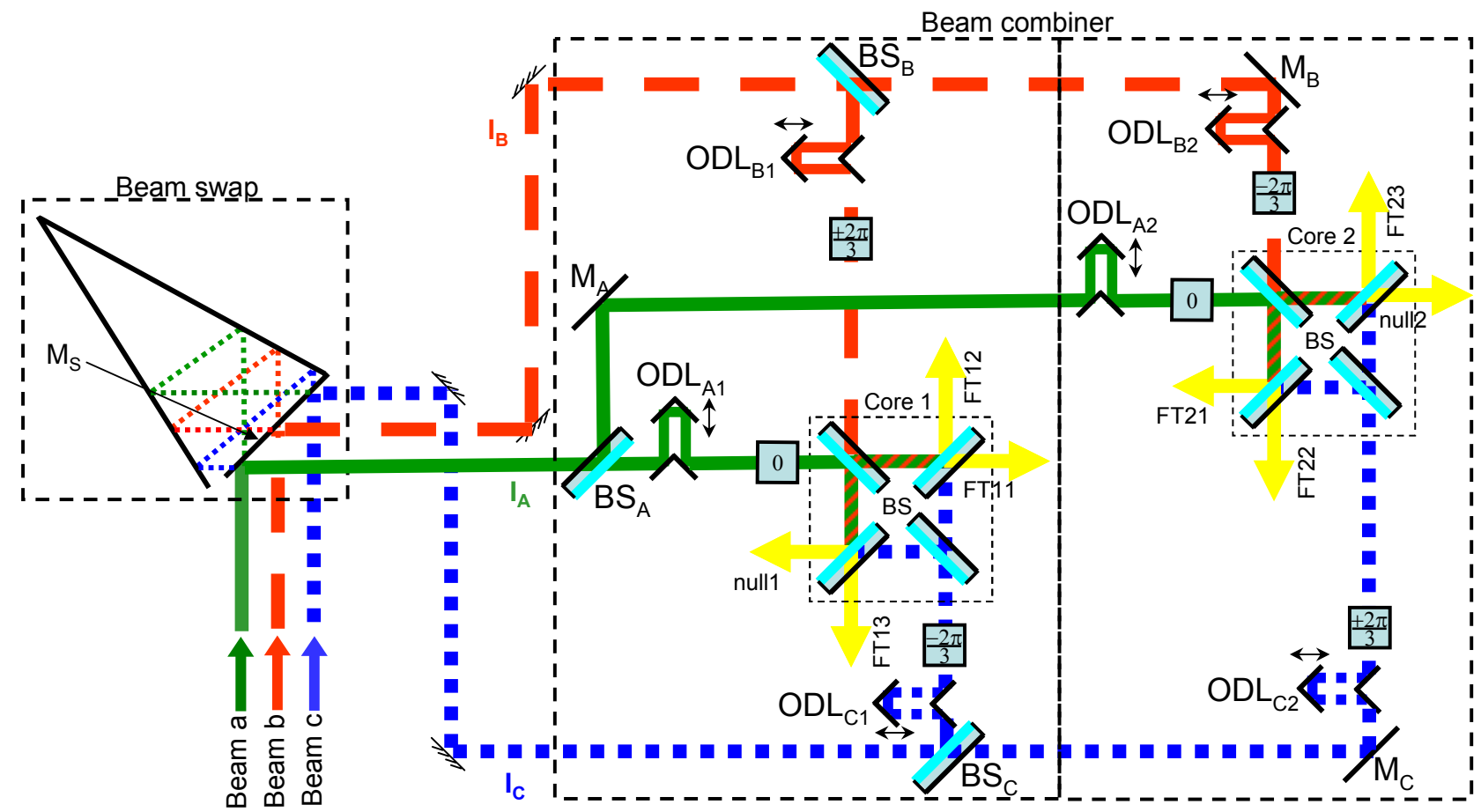

Figure 6: Schematic of the proposed nulling/imaging concept breadboard based on the TNO BC.

Two of the beams can be changed order by flipping mirror $\mathrm{M}_{\mathrm{S}}$ out of the path of the three beams. This redirects the three beams to the beam swap optics, swapping beams A and C, leaving beam B unaffected. 


\section{CONCLUSIONS}

From the trade-off study it is concluded that the TNO concept is the most feasible concept since it is the only concept capable of implementing end-to-end FT. The downside of this concept is its low modulation efficiency. However, it is anticipated that there is scope to significantly improve upon this figure and further work will be done to improve the modulation efficiency of the TNO concept. The next goal is to test the feasibility of the TNO concept with a breadboard design.

\section{ACKNOWLEDGMENTS}

The authors would like to thank the NIVR for support of this study.

\section{REFERENCES}

1. ESA report, "Darwin - Mission Requirements Document," SCI-A/2005/287/Darwin/DMS, 2005.

2. L. d'Arcio and R. Le Poole, "Beam Combiner Concept for the Darwin Imaging Mode," Proc. of the SPIE SP-539, 2003.

3. A. Karlsson, L. Kaltenegger, R. den Hartog, L. d'Arcio, M. Kilter, Ch. Erd, and F. Ankersen, "Darwin, TTN+ Mission Design Assessment," ESA Communication SCI-A/2004/287/Darwin/DMS, pp. 39, 2004. 